\title{
Estudio cualitativo en un grupo de estudiantes ourensanos/as sobre el fenómeno del Sexting
}

\author{
Patricia Alonso-Ruido*, Yolanda Rodríguez-Castro*, Carmen Pérez-André*, María José-Magalhães ** \\ *Facultad de Ciencias de la Educación, Universidad de Vigo (España) \\ ** Faculdade de Psicologia e de Ciências da Educação, Universidade do Porto (Portugal)
}

\begin{abstract}
Resumen
El Sexting es el envío, recepción y reenvío de fotografías, vídeos y/o textos de tipo erótico-sexual, a otras personas a través de teléfonos móviles, tablets, redes sociales u otros medios informáticos. El objetivo de esta investigación consistió en identificar comportamientos, motivaciones y percepciones de los/as adolescentes sobre el Sexting. La muestra está formada por 47 adolescentes de la ciudad de Ourense, con un rango de edad de 15 a 18 años. A través de una metodología cualitativa, se observa que inicialmente la tónica general era no admitir ninguna práctica de sexting, no obstante sí admitían que es algo frecuente entre la gente de su edad, alegando motivaciones para practicarlo múltiples y variadas.

Palabras clave: sexting, comportamientos, motivaciones, educación.
\end{abstract}

La revolución digital a la que asistimos en los últimos años ha supuesto drásticos cambios en las formas de comunicación y socialización en las sociedades occidentales (Aguirre, Zavariz, \& Casco, 2012). Estos cambios están relacionados fundamentalmente con el uso generalizado de la telefonía móvil, que nos permite una comunicación cada vez más fácil e inmediata (Fundación Telefónica, 2009). De esta manera se ha modificado la forma en la que conectamos unos/as con otros/as (Cruz \& Soriano, 2014). Eliminándose las formas sociales tradicionales, que ya no pueden servir como marco de referencia para las acciones humanas, generándose así la sociedad Red, donde la comunidad adquiere un matiz de conexiones y desconexiones aleatorias (Bauman, 2001; Castell, 2001).

En este marco de conexiones/desconexiones virtuales conviven dos generaciones, la generación "before computer” (Freixa, 2006) y aquella que ha nacido y crecido interactuando con diversos dispositivos tecnológicos, los/as "nativos/as digitales". Ambas generaciones hacen un acceso y uso de la Red fundamentalmente intuitivo y espontáneo (Gutiérrez, 2014), generalmente sin formación/información de los posibles peligros a los que se exponen.

Es precisamente en este marco de virtualidad, caracterizado por la generalización del uso de la telefonía móvil, la mensajería instantánea o las redes sociales en donde surgen nuevas prácticas de riesgo virtual y real, como el Sexting.

El objetivo,de esta investigación consiste en identificar los comportamientos, motivaciones y percepciones de los/las adolescentes de la ciudad de Ourense sobre el Sexting.

\section{Delimitación del fenómeno}

El vocablo Sexting es un término anglosajón que resulta de la combinación de sex (sexo) y texting (envío de mensajes de texto). Las primeras aportaciones a la temática partían de una visión restrictiva, limitándolo al envío de mensajes de texto (OSI, 2011). Posteriormente en base a las aportaciones de diferentes investigaciones sobre el fenómeno (Ferguson, 2011; Lenhart, 2009; Weisskirch \& Delevi, 2011) y a la rápida evolución de los dispositivos tecnológicos (Martínez, 2013), se amplía la definición incluyendo fotografías, vídeos y/o textos.

De esta forma la conceptualización de partida más abarcadora conceptualiza el fenómeno como el envío, recepción y reenvío de contenidos de tipo erótico-sexual, sean fotografías, vídeos o textos, a otras personas a través de teléfonos móviles, tablets, redes sociales u otros medios informáticos.

\section{Prevalencia nacional e internacional}

En el ámbito internacional las tasas de prevalencia en adolescentes son muy variadas. Entre los 10 y los 19 años aquellos estudios que se refieren al envío o recepción de fotografías erótico sexuales revelan cifras situadas en una horquilla del 2.5\% y el 41\% (AP-MTV. 2009; Hinduja \& Patchin, 2010; Kopecky, 2011; Mitchell, Finkelhor, Jones \& Wolak, 2012; NCPTUP, 2008; Strassber, Mckinnion, Sustaíta \& Rullo, 2013; Temple et al., 2012). Por lo que debido a este gran abanico de cifras, no podemos estimar el alcance real del fenómeno. No obstante, sí se evidencia que es una práctica común entre los adolescentes.

Relativo al ámbito nacional todavía no existen evidencias suficientes en referencia a esta práctica entre los/as adolescentes (Martínez \& Boo, 2014; Agustina, 2010). Una primera aproximación al estudio del fenómeno, es la investigación realizada en 322 adolescentes españoles/as por INTECO (2009). Proporciona cifras relativas al sexting pasivo y activo, situando la práctica entre el $8.1 \%$ y $4 \%$ respectivamente.

Posteriormente la investigación de Fajardo, Gordillo \& Regalado (2013) que analiza este fenómeno en una muestra de 132, revela que los/as adolescentes no reconocen el envío de fotos o vídeos sexys de sí mismo/las, a la par que sí afirman conocer a personas que se ha sentido acosadas/incomodadas por la Red. También muestran en sus resultados que los/as adolescentes entienden el Sexting como una forma de pornografía $(65,9 \%)$ o de acoso sexual (26,52\%). 
Más recientemente en un estudio realizado en Galicia se muestra que el $4 \%$ de los/as adolescentes afirman enviar a otras personas fotos o vídeos de sí mismo/las con contenido erótico/ sexual a través de Internet (Defensor del Pueblo, 2014).

A nivel cualitativo existen muy pocos estudios (Walker, Sanci \& Temple, 2013; Ringrose, Harvey, Gill \& Livingstone, 2013; NSPCC, 2012; Torres, 2014) que se centren en evaluar tanto el impacto del fenómeno como de sus consecuencias. No obstante, arrojan al estudio del sexting resultados muy interesantes, por ejemplo que el sexting es una actividad influenciada por las dinámicas de género, la existencia del doble rasero en torno a la sexualidad y las prácticas de sexting, la presión o coacción como motivación para la práctica, que las chicas se ven afectadas más negativamente de las consecuencias de esta actividad o que es una práctica relacionada con la cultura.

A pesar de existir cifras tan dispares en torno a la práctica de sexting, se demuestra que este tipo de comportamientos son realizados por las/os adolescentes de forma habitual con mayor o menor frecuencia exponiéndolos/as a ciertos peligro. Tal y como han demostrado estudios recientes existe relación directa entre esta práctica y la discriminación (bullying) (Bosman, Bayraktar \& d'Haenens, 2015) o incluso que los/as adolescentes que reportaron recibir sexts muestran mayor riesgo de ser acosadores/as cibernéticos (Wachs, Junger \& Sittichai, 2015).

\section{Método}

Participantes

Se eligieron dos centros de Educación Secundaria Obligatoria de la ciudad de Ourense y dentro de los mismos se seleccionaron al azar aulas relativas a $3^{\circ}, 4^{\circ} \mathrm{y}$ $1^{\circ}$ de Bachillerato. De forma que en el presente estudio se muestras los resultados de cuatro grupos de discusión, dos de $3^{\circ}$ de E.S.O., uno de $4^{\circ}$ y un último grupo de $1^{\circ}$ de Bachillerato. La muestra quedó formada por 46 adolescentes con un rango de edad de los 15 a los 18 años. De los cuales 22 son chicas y 25 chicos.

Para la realización del cuestionario se solicitó la autorización de los padres y madres, de los y las estudiantes participantes en el estudio.

\section{Instrumentos}

El diseño de la investigación se basa en una metodología cualitativa. Se utilizó como instrumento de investigación los grupos de discusión o Focus Groups. Técnica basada en la conversación socializada en la que la comunicación grupal sirve para captar los discursos ideológicos y las representaciones simbólicas asociadas a los fenómenos sociales (Arboleda, 2008).

A través de un guion de preguntas de carácter semi-estructurado se les preguntaba sobre sus comportamientos en relación a la creación y difusión de contenidos de sexting. Así como la percepción sobre la frecuencia de este tipo de comportamientos entre la gente de su edad. También se les preguntaba sí conocían casos cercanos de esta práctica y cuáles eran las principales motivos por lo que tanto ellos/as como la gente de su edad practicaba sexting.

\section{Procedimiento}

Para realizar los grupos de discusión, las clases se dividieron en dos grupos más o menos heterogéneos, procurando la representación equilibrada de ambos géneros. Las sesiones fueron grabadas a través de dispositivos de recogida de voz por una investigadora. Se desarrollaron en el propio centro académico, con una duración de 40-50 minutos y dentro del horario lectivo.

\section{Análisis de la información}

Para analizar la información obtenida se utilizó la técnica de análisis de contenido naturalista (Bardin, 1986).

Por motivos de confidencialidad y rigor científico para preservar la identidad de cada participante, se asignó a cada uno/a un código que significa GD (grupo de discusión), 1, 2, 3 o 4 (el número del grupo correspondiente), chico/chica (género de cada alumno o alumna), seguido del número del o de la alumna que verbaliza dicho comentario y finalmente l. (línea) relativo a la línea en la que se encuentra cada expresión en la transcripción literal.

\section{Resultados}

En referencia a la práctica de sexting, mediante el envío o publicación en redes sociales de fotografías suyas erótico/sexuales, a pesar de que mayoritariamente negaban este tipo de práctica, había chicos y chicas que admitían abiertamente realizarla: "Si tengo" (GD1, Chica4, l.86); "En la playa si (foto biquini posición erótico-sexual)” (GD1, Chica2, l.88).

En algunos casos explicaban que sí lo habían hecho pero por determinadas causas como:

"Igual si estaba bajo los efectos del alcohol”. (GD3, Chico6, 1.121)

"Yo sí pero en plan broma. Para mandárselas aquel chico. Porque me hacía gracias.” (GD4, Chico6, l. 122-123)

"Tenemos un grupo de whatsapp de 6 o 7 amigos que nos mandamos fotos así de coña y videos. Pero son fotos graciosas no tienen nada que ver con esto" (GD4, Chico2, l. 124-125).

No obstante, en algunos casos no consideraban estas prácticas sexting:

"Yo subo una foto así. [foto contra el espejo sin camiseta]. No lo considero sexting” (GD1, Chico3, 1.82)

Por otro lado en referencia a la recepción de fotografías erótico/sexuales de otras personas prácticamente la totalidad de los/as adolescentes respondían que sí recibían este tipo de contenidos con mucha frecuencia, tanto de personas conocidas como desconocidas, alegando que:

“Te mandan de todo. [WhatsApp]" (GD1, Chico 2, 1. 114)

"Tú entra en los twitter a ver lo que te encuentras" (GD2, Chica2, l.125)

"Y en cantidad" (GD3, Chico1, 1. 171).

Cuando se les pregunta que hacen con esas imágenes cuando están en su poder, afirman de forma mayoritaria que las eliminan: 
“Las elimino si me llegan” (GD1, Chica 4, 1.95)

“La coges y la borras” (GD1, Chica 6, l.105)

Otros sin embargo, si relatan que hay compañeros/as que exponen y difunden estas fotografías

"A mí me mandaron hace tiempo unas fotos de chicas que conocía, que son amigos que te las van pasando y se ríen, por whatsapp” (GD2, Chico2, 1.100-101).

En lo relativo a sí creen que es frecuente que la gente de su edad se haga fotos erótico/sexuales y las difundas (RS o WasthApp) de forma mayoritaria en los grupos aseguran que sí, relatando casos en su entorno de prácticas de sexting entre la gente de su edad:

"Yo en ese grupo no pero sí que en otros grupos que te dicen: Pues mira que foto me enviaron (erótica o sexual)" (GD1, Chica 6, 103-104)

"Un chico de nuestra clase colgó unas fotos de una chica de clase desnuda, de aquellas se usaba tuenti y hubo problemas y tal” (GD3, Chica1, l.158-159).

"Yo sí, posando desnudas y de gente conocida" (GD4, Chico6, l.141).

En lo relativo a la práctica de sexting mediante la creación, envío, intercambio y/o difusión de vídeos erótico-sexuales, la mayoría de los y las adolescentes refieren no realizarlo, incluso uno de los chicos afirma que: "Yo creo que es más frecuente las fotos que los vídeos supongo" (GD4, Chica3, l.168). No obstante sí admiten recibir este tipo de contenidos de otras personas, afirmando que los/as adolescente lo hacen y lo difunden, explicando que: "A mí de mandarme un enlace de un video de aquí de Ourense. Salía el chico bailando sin ropa” (GD1, Chico3, l.134-135); “Uno se grabó con su novia y lo subió a una página porno. Y me mandaron el enlace” (GD1, Chica2, l.136-137).

No obstante, uno de ellos se excusa refiriendo que es lo normal tener este tipo de contenidos: "Pero yo tengo algún video en el móvil, no es por ser así un guarro pero..." (GD4, Chico2, l.167).

En lo relativo al envío o difusión de textos erótico-sexuales, inicialmente tampoco admitían enviar textos erótico-sexuales, no obstante algunos y algunas sí refieren hacerlo, incluso afirman que es Lo lógico (GD3, Chico6, 1. 207) o De toda la vida (GD3, Chico5, l. 208) y explican que estos textos son: “¿Quedamos y tal? Y ellas dicen ¿Para qué? Y decimos pues ya sabes para qué. Tengo que explicártelo o algo, te hago señas ahí” (GD3, Chico5, l. 209-210) y "Claro esas las caritas de guarrillo (emoticono)” (GD3, Chico6, l.211).

Por último en lo tocante a los motivos que aluden tanto ello y ellas para el envío de estos contenidos, como los que creen que tiene la gente que práctica sexting, refieren causas múltiples y variadas como:

"Para llamar la atención” (GD1, Chica2, l. 148)

"Para que le hagan caso” (GD1, Chica1, l. 149)

"Para excitar a la otra persona” (FG1, Chico3, l.121)

"Se aburren” (GD2, Chico2, l. 144)

"Para tener fama" (GD2, Chico4, l.135)

"Para presumir, para hacerse el guay” (GD2, Chica5, 1.137)

Entre los motivos destacan, por un lado, las influencias sociales o la presión del grupo para practicarlo pues expresan causas como: "Está de moda” (GD2, Chica6,
1.138); "Es influencia de las película porque ves como disfrutan los actores entonces la gente se pone así” (GD2, Chica4, 1.142-143); e incluso "Lo hacen porque una amiga lo hizo y le dice pues no eres capaz de hacerlo y van y lo hacen” (GD2, Chica1, l.145-146).

Por otro lado, refieren también el anonimato y la protección de los dispositivos electrónicos como causa para realizar sexting, verbalizando que: "Porque no se atreve a decirlo a cara” (GD3, Chico5, l.215); "Yo creo que [es porque] por whatsapp se pierde más la vergüenza” (GD4, Chica1, l.190).

Por último, en algunos casos aludían a la inconsciencia de las consecuencias de este tipo de prácticas expresando que los y las adolescentes lo hacen: "Porque no tienen dos dedos de frente" (GD1, Chico2, 1.119) y que "Es que hay que ser tonto para difundir eso" (GD4, Chico3, 1.171).

\section{Discusión}

En referencia al comportamiento en sexting, hemos encontrado que de forma general los/as adolescentes inicialmente no reconocen su participación activa en este comportamiento. Estos resultados van en la línea de los revelados en diversas investigaciones nacionales e internacionales que situaban prevalencias baja, no alarmistas (Finkelhor, 2014), pero que visibilizan esta práctica entre los y las adolescentes (Hinduja \& Patchin, 2010; Mitchell, Finkerhor, Jones, \& Wolak, 2012). Nuestros resultados también se sitúan en la línea de los reveledados por Fajardo, Gordillo \& Regalado (2013) que muestran en su estudio que los/as adolescentes no reconocen su participación en actos de sexting. Expresando que no es muy frecuente que las personas de su edad envíen mensajes (el 37.14\% así lo considera) ni fotos y vídeos sexys a alguien en el 51.52\% de los casos.

Nuestros resultados al igual que otros estudios anteriores (Flescheler Peskin et al., 2013; Lenhart, 2009; Strassberg, McKinnon, Sustaíta \& Rullo, 2013) también evidencian que los/as adolescentes admiten en mayor medida la recepción de sexts que la producción y difusión personal.

Hemos encontrado cierto desconocimiento del fenómeno del sexting, dado que en algunos casos no eran capaces de identificar determinadas prácticas como sexting. Tal y como muestran Walker, Sanci \& Temple, (2013) se evidencia que a pesar de que la práctica es ampliamente conocida, existe cierto desconocimiento a nivel teórico.

Observamos que de forma general en nuestro estudio, cuando las chicas y los chicos relatan situaciones de sexting, suelen hacerlo en femenino, por lo que podríamos afirmar que son las chicas las que más lo practican. No obstante, los estudios realizados hasta el momento no muestran resultados concluyentes en cuanto al género (Klettke, Hallford \& Mellor, 2014).

En lo tocante a las motivaciones, las causas que aludían son múltiples y similares a las refflejadas en otros estudios. Por ejemplo, el aburrimiento o captar la atención de la persona emisora y tener relaciones íntimas con ella, son motivos revelados por varias investigaciones (see also, Kopecký, 2012; Lenhart, 2009; 
Livingstone \& Smith, 2014). No obstante, nuestros resultados evidencian que la presión del grupo o de la pareja puede ser un motivo para enviar fotografías, vídeos o mensajes erótico-sexuales entre los/as adolescentes, tesis apoyada en numerosos estudios (AP-MTV, 2009; Henderson \& Morgan, 2001 Englander, 2012; NCPTUP, 2008).

Por último, los discursos de los/as adolescentes muestran la importancia del anonimato a la hora de realizar este tipo de practicas. Tal y como expresaba Döring (2000) en la Red se mantienen ciertas actitudes que en la vida off line no se realizarían.

En conclusión por todo lo relatado el estudio del fenómeno del Sexting así como los riesgos y consecuencias que este tipo de prácticas lleva parejos resulta un ámbito de estudio y análisis necesario. Con el objetivo de formar y concienciar sobre el sexting a los/las adolescentes educando en la prevención de los riesgos que la práctica de sexting tiene asociados, pero también al conjunto de profesionales, docentes y familias para así ofrecer la adecuada respuesta psicosocioeducativa.

\section{Referencias}

Aguirre, P., Zavaríz, A., \& Casco, J. (2012). El Sexting ¿Exhibición o Violencia Simbólica en los Jóvenes?: Sexting, lo público, lo privado y lo íntimo de una práctica juvenil. Alemania: Editorial Académica Española.

Agustina, J. (2010). ¿Menores infractores o víctimas de pornografía infantil? Respuestas legales e hipótesis criminológicas ante el Sexting. Revista Electrónica de Ciencia Penal y Criminología, 12-11, 1-44.

Arboleda, L. M. (2008). El grupo de discusión como aproximación metodológica en investigaciones cualitativas. Revista Facultad Nacional Salud Pública, 26 (1), 69-77.

Associated Press \& MTV. (2009). MTV Digital Abuse Survey, Executive Summary. Recuperado de http://www.athinline.org/pdfs/MTV-AP_2011_Resear ch_Study-Exec_Summary.pdf.

Bardin, L. (1986). Análisis de contenido. Madrid: Akal

Bauman, Z. (2001). La globalización: consecuencias humanas. México: Fondo de Cultura Económica.

Bosman, J., Bayraktar, F., \& D'Haenens, L. (2015). Children's Digital Media Practices within the European Family Home: Does Perceived Discrimination Matter?. Journal of Children and Media, 9(1), 77-94. doi: 10.1080/17482798.2015.997099.

Castell, M. (2001). La Era de la Información. Vol. II: El poder de la identidad. México, Distrito Federal: Siglo XXI Editores.

Cruz, L., \&Soriano, E. (2014). Psychological Aspects, Attitudes and Behaviour Related to the Practice of Sexting: A Systematic Review of the Existent Literature. Procedia - Social and Behavioral Sciences, 132, 114-120. doi:10.1016/j.sbspro.2014.04.286.

Englander, E. (2012). Low risk associated with most teenage sexting: A study of 617 18-year-olds. Disponible

en http://webhost.bridgew.edu/marc/SEXTING\%20 AND\%20COERCION\%20report.pdf.
Fajardo, M. I., Gordillo, M., \& Regalado, A. B. (2013). Sexting: Nuevos usos de la tecnología y la sexualidad en adolescentes. International Journal of Developmental and Educational Psychology, 1(1), 521-534.

Feixa, C. (2006). Generación XX. Teorías sobre la juventud en la era contemporánea. Revista Latinoamericana de Ciencias Sociales, Niñez y Juventud, 4(2). Universidad de Manizales.

Ferguson, C. J. (2011). Sexting behaviors among young hispanic women: Incidence and association with other high-risk sexual behaviors. Psychiatric Quarterly, 82(3), 239-243. doi: 10.1007/s11126-010-9165-8.

Finkelhor D. (2014). Commentary: cause for alarm? Youth and internet risk research, a commentary on Livingstone and Smith. Journal of Child Psychology and Psychiatry, 55(6), 655-658. doi:10.1111/jcpp.12260.

Fleschler-Peskin, M., Markham, C. M., Addy, R. C., Shegog, R., Thiel, M., \& Tortolero, S. R.(2013). Prevalence and patterns of sexting among ethnic minority urban high school students. Cyberpsychology, Behavior and Social Networking, 16(6), 454-459. doi:10.1089/cyber.2012.0452.

Fundación Telefónica. (2009). La Generación Interactiva en España. Niños y adolescentes ante las pantallas. Ariel. Recuperado de http://www.generacionesinteractivas.org/?page_id=16 78.

Gutiérrez Morales, I. M. (2014). Cyberbullying y sexting: percepción y propuestas de estudiantes universitarios. Revista Multidisciplina, 17(1), 93-119.

Henderson, L., \& Morgan, E. (2011). Sexting and sexual relationships among teens and young adults. McNair Scholars Research Journal, 7(1), 31-39.

Hinduja, S. \& Patchin, J. W. (2010). Sexting: A brief guide for educators and parents. Recuperado de http://www.cyberbullying.us/Sexting_Fact_Sheet.pdf.

Klettke , B., Hallford, D., \& Mellor, D. (2014). Sexting prevalence and correlates: A systematic literature review. Clinical Psychology Review, 34(1), 44-53. doi: 10.1016/j.cpr.2013.10.007.

Kopecký, K. (2011). Sexting among Czech preadolescents and adolescents. New Educational Review. 28(2), 39-48.

Lenhart, A. (2009). Teens and sexting: How and why minor teens are sending sexually suggestive nude or nearly nude images via text messaging. Recuperado de:

http://www.pewinternet.org/files/oldmedia/Files/Repo rts/2009/PIP_Teens_and_Sexting.pdf.

Livingstone, S., \& Smith,K. (2014). Anual Research Review: Harms experienced by child users of online and mobile technologies: the nature, prevalence andmanagement of sexual and aggressive risks in the digital age. Journal of Child Psychology and Psychiatry, 55(6), 635-654. doi: 10.1111/jcpp.12197.

Martínez Otero, J. (2013). La difusión de sexting sin consentimiento del protagonista: un análisis jurídico. Derecom. Nueva Época, 12(2), 1-16.

Martínez Otero, J., \& Boo Gordillo, A. (2012). El fenómeno del sexting en la adolescencia: descripción, 
riesgos que comporta y respuestas jurídicas. En J. García González (Eds.), La violencia de género en la adolescencia (pp. 289-323). Navarra: Aranzadi.

Mitchell, K. J., Finkerhor, D., Jones, L. M., \& Wolak, J. (2012). Prevalence and characteristics of youth sexting: A national study. Pediatrics, 129, 13-20. doi:10.1542/peds.2011-1730.

Observatorio de la Seguridad de la Información. (2011). Guía sobre adolescencia y sexting: qué es y cómo prevenirlo. Madrid: Instituto Nacional de Tecnologías de la Comunicación.

Ringrose, J., Gill, R., Livingstone, S., \& Harvey, L. (2012). A Qualitative Study of Children, Young People and 'Sexting'. London: NSPCC. Disponible en http://www.nspcc.org.uk/globalassets/documents/rese arch-reports/qualitative-study-children-young-peoplesexting-report.pdf.

Ringrose, J., Harvey, L., Gill, R., \& Livingstone, S. (2013). Teen girls, sexual double standards and 'sexting': Gendered value in digital image exchange. Feminist Theory, 14(3), 305-323. doi: 10.1177/1464700113499853.

Strassberg, D. S., McKinnon, R. K., Sustaita, M. A., \& Rullo, J. (2013). Sexting by high school students: An exploratory and descriptive study. Archives of Sexual Behavior, 42(1), 15-21.doi: 10.1007/s10508-012-9969-8.

Temple, J. R., Paul, J. A., Van den Berg, P., Le, V. D., McElhany, A., \& Temple, B. W. (2012). Teen sexting and its associations with sexual behaviours. Archives of Pediatrics and Adolescent Medicine, 166(9), 828$833 . \quad$ http://dx.doi.org/ 10.1001/archpediatrics.2012.835.

The National Campaign to Prevent Teen and Unplanned Pregnancy. (2008). Sex and Tech: results from a survey of teens and young adults. Recuperado de. http://www.thenationalcampaign.org/sextech/PDF/ SexTech_Summary.pdf.
Torres, G. (2014). Uso de las redes sociales virtuales en un grupo de estudiantes de un colegio femenino privado en Santiago de Chile: sus implicaciones psicológicas y algunos aportes sobre el tema. Itinerario Educativo, (64), 143-161.

Valedor do Pobo de Galicia. (2014). Informe Adolescentes e Internet en Galicia: "Mocidade on line" en Galicia. Recuperado de http://descargas.valedordopobo.com/index.php?s= $156 \& \mathrm{i}=136$.

Wachs, S., Junger, M., \& Sittichai, R. (2015). Traditional, Cyber and Combined Bullying Roles: Differences in Risky Online and Offline Activities. Societies, 5(1), 109-135. doi:10.3390/soc5010109

Walker, S., Sanci, L., \& Temple-Smith, M. (2013). Sexting: Young womens and mens's views on its nature and origins. Journal of Adolescent Health, 52(6), 697-701. doi: 10.1016/j.jadohealth.2013.01.02.

Weisskirch, R., \& Delevi, R. (2011). "'Sexting' and adult romantic attachment. Computers in Human Behavior. 27(5), 1697-1701. doi:10.1016/j.chb.2011.02 